\title{
Usefulness of Cone Beam Intra-Arterial CTA for Evaluation of Flow Diverters: A Practical Approach for Daily Use
}

\author{
Dennys Reyes Victor Becerra Indiana Alcala Italo Linfante \\ Guilherme Dabus \\ Division of Neurointerventional Surgery, Miami Cardiac \& Vascular Institute and \\ Baptist Neuroscience Center, Miami, FL, USA
}

\author{
Keywords \\ Flow diverter $\cdot$ Cone beam computed tomography $\cdot$ Cerebral aneurysm
}

\begin{abstract}
Cone beam computed tomography $(\mathrm{CBCT})$, initially used for evaluation of intraprocedural complications such as hemorrhage, has evolved to provide details of implanted devices such as flow diverters. The study aim is to present our experience in using CBCT with intra-arterial injection and provide a step-by-step approach for postprocessing in a practical protocol for daily use. IRB approval was obtained, and the neurointerventional database was retrospectively reviewed from July 2012 to June 2017. Patients who underwent cone beam intra-arterial CT angiography for evaluation of implanted flow diverter devices were reviewed. Patient demographics, aneurysm location (internal carotid artery [ICA]-cavernous, ICA-paraclinoid, and ICA-distal; middle cerebral artery [MCA], anterior cerebral artery [ACA]-acom, ACA-pericallosal, vertebral artery [VA]), type (saccular, dissecting fusiform, or blister) and size, device, injection technique (contrast dilution, rate, and volume), and reconstruction protocol were recorded. Acquired images were postprocessed using a Philips Xtravision workstation. Eighty patients ( 63 women and 17 men) met the inclusion criteria of our study. Age range was $25-80$ years old. Treated aneurysms were located in the ICA-paraclinoid in 48 cases (60\%), ICA-distal in 12 cases, ICA-cavernous in 8 cases, MCA in 4 cases, VA in 4 cases, ACA-acom in 2 cases, ACA-pericallosal in 2 cases; 69 were saccular, 8 fusiform, and 3 ruptured blister aneurysms. There were 52 small, 20 large, and 8 giant aneurysms. Pipeline (Medtronic, MN, USA) was the predominant device used in 77 procedures. Two injection techniques were used: $2.5 \mathrm{~mL} / \mathrm{s}$ for
\end{abstract}


a total volume of $55 \mathrm{~mL}$ with a 2-s imaging delay or $3 \mathrm{~mL} / \mathrm{s}$ for a total volume of $70 \mathrm{~mL}$ with a 3 -s imaging delay; contrast (loxilan $300 \mathrm{mgl} / \mathrm{mL}$ ) dilution was $10-20 \%$ in all cases. The device's landing zones, conformability, presence of deformities, and wall apposition were successfully visualized in all cases. Metal artifact reduction program was applied in 9 coiled aneurysms, and this was satisfactory as well.

(c) 2018 S. Karger AG, Basel

\section{Introduction}

The use of flow diverters for the treatment of intracranial aneurysms continues to evolve. They represent an effective alternative treatment for some types of aneurysms by redirecting blood flow into the parent vessel leading to occlusion of the aneurysm and finally vessel remodeling [1, 2]. Flow diverters create a favorable environment for aneurysmal thrombosis by creating an interface between the artery and the aneurysm with a disrupting mechanism of the inflow and outflow within the saccular aneurysm, and gradually resulting in aneurysmal occlusion [2,3].

Radiological evaluation of flow diverter braid expansion and conformability to the vessel wall has become useful in preventing complications carrying important clinical applicability $[3,4]$. Common scenarios such as incomplete coverage of an aneurysm neck, kinking, or incomplete expansion and malapposition of a stent carry a significant risk for thromboembolic events [3,4]. Conventional cerebral angiography (digital subtraction angiography, DSA), CT angiography (CTA), and magnetic resonance angiography (MRA) of the head techniques have been applied for intra- and postprocedural visualization of flow diverters; however, these techniques have limitations $[3,5,6]$. The use of cone beam computed tomography (CBCT) imaging during neurointerventional procedures has been increasing rapidly. Initially used as a CT scan for evaluation of intraprocedural complications such as hemorrhage, it has evolved to provide details of implanted devices such as flow diverters. This imaging modality has been used to provide meaningful radiological information with regard to the neurovascular anatomy and morphology as well as implanted devices $[1,4,7]$.

The aim of this study is to present our experience in using cone beam CTA with intraarterial injection for evaluation of implanted flow diverters demonstrating its applicability in a practical protocol for daily use.

\section{Methods}

Subject Selection

After IRB approval was obtained, the neurointerventional database from our institution was retrospectively reviewed from July 2012 to June 2017. Patients who underwent endovascular therapy of cerebral aneurysm with flow diverter devices followed by completion of a cone beam intra-arterial CTA (CBCT) for qualitative visualization of the device in relation to the host vessel were included in the analysis.

Imaging Protocol Including 3D Image Analysis

All endovascular procedures were performed under general anesthesia using a biplane flat panel detector neuroangiographic equipment (Philips Allure FD20/10 or FD20/15). The area of interest was positioned in the center of rotation, with the catheter previously in place within the ipsilateral internal carotid artery (ICA) or the vertebral artery (VA). The acquisition was obtained using the CBCT ICS 22 algorithm protocol available in our equipment. Two contrast injection techniques were used: a $2.5 \mathrm{~mL} / \mathrm{s}$ for a total volume of $55 \mathrm{~mL}$ with a 2-s imaging delay, and a $3 \mathrm{~mL} / \mathrm{s}$ for a total volume of $70 \mathrm{~mL}$ with a 3-s imaging delay. The injection protocol was decided depending on the location of the catheter. The concentration of the contrast Ioxilan with $300 \mathrm{mgI} / \mathrm{mL}$ included a dilution formula of $10-20 \%$ of the contrast. The acquired 
images were postprocessed using our standardized protocol through the Philips Xtravision workstation. An automated metal artifact reduction function was selected when coils were used.

For this study, the recorded data included patient's demographics, vascular territory involved and morphology of the aneurysm. The recorded vascular segments were defined as ICA cavernous, paraclinoid, and distal; middle cerebral artery (MCA), anterior cerebral artery (ACA) anterior communicating artery and pericallosal; and VA. An experienced operator (G.D.) qualitatively evaluated the device's landing zones, conformability to the arterial geometry, presence of deformities, and wall apposition to determine whether the generated images were critically visualized or not. A descriptive analysis of the data was utilized with attention to the technical results, and a review of the available literature was included in this study.

\section{Results}

Of 160 aneurysms treated with flow diverter in our institution, 80 patients met the inclusion criteria for our study. Patients' ages ranged from 25 to 80 years old. There were a total of 63 women (79\%) and 17 men (21\%). The most commonly treated aneurysms were located in the ICA-paraclinoid segment (48 cases, 60\%). The remaining aneurysm were located in the ICA-distal in 12 cases, ICA-cavernous in 8 cases, MCA in 4 cases, VA in 4 cases, ACA-Acom in 2 cases, ACA-pericallosal in 2 cases. $95 \%$ of the cases were located in the anterior circulation. A total of 69 treated aneurysms were saccular (86\%), 8 fusiform (10\%), and 3 ruptured blister (4\%) aneurysms. There were 52 small (65\%), 20 large (25\%), and 8 giant $(10 \%)$ aneurysms. The Pipeline embolization device (Medtronic, MN, USA) was the most commonly used in our cohort for a total of 77 procedures (96\%).

CBCT accurately demonstrated the landing zones and conformability to the arterial geometry and aneurysm neck in all cases. The presence of deformity was seen in one case, and significant wall malapposition was visualized in 2 cases at the end of the procedure. In previously coiled aneurysms (12 cases, 15\%), the metal artifact reduction function was felt to enable satisfactorily the evaluation of the implanted devices.

\section{Discussion}

Our study demonstrates the practical applicability of cone beam CTA with intra-arterial injection for evaluation of implanted flow diverters.

Currently, evaluation of intracranial implantable devices, including flow diverters and self-expanding stents, often involves intra- and postprocedural visualization using DSA and conventional CT/MR angiography techniques $[4,6,8]$. As these devices become more tailored for use in the neurovasculature, certain qualities to adapt to the small and circuitous nature of intracranial vessels in order to achieve optimal outcomes are required [4]. Some of these qualities include increased flexibility, smaller size, appropriate radiopacity, and adequate radial strength $[6,8]$. Visualization of neurovascular stents with DSA can be challenging given the low radio-opacity quality [3-5]. Flow diverters are stent-like devices characterized by low porosity with high pore density. Two different flow diverters were used during this study, Pipeline embolization device (Medtronic) or a Surpass neuroendograft device (Stryker, MI, USA) [9, 10]. The Pipeline embolization device (Medtronic) is composed of $75 \%$ cobalt chromium and $25 \%$ platinum tungsten, whereas the Surpass neuroendograft device (Stryker) is composed of 92\% platinum and 8\% tungsten with cobalt chromium alloy [10]. The Surpass device (Stryker) is a 72-wire braid, whereas the Pipeline embolization device (Medtronic) is a 48-strand braided mesh. In our study, the two stents were equally well visualized. 

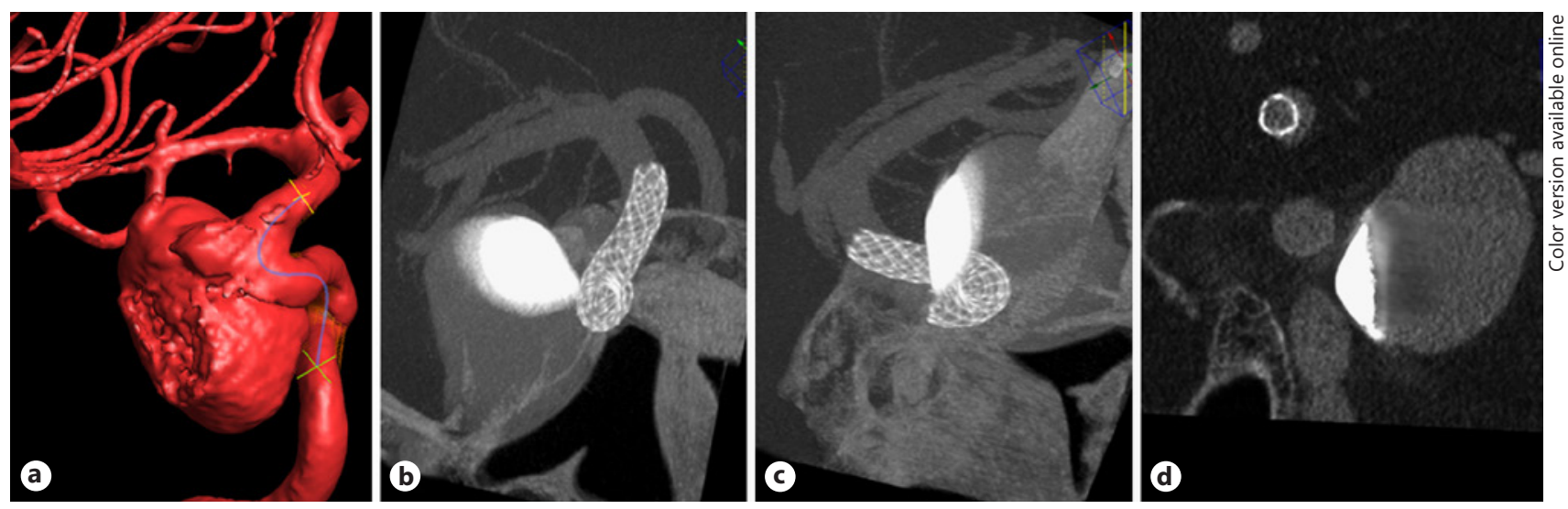

Fig. 1. a Case of a large paraclinoid transitional aneurysm treated with a Surpass (Stryker) flow diverter. b-d Reconstruction of IA Vaso-CT (cross-sectional) demonstrates malapposition of the distal end of the device due to the angle between the end of the device and the vessel curvature. Notice the stagnation of contrast in the aneurysm.
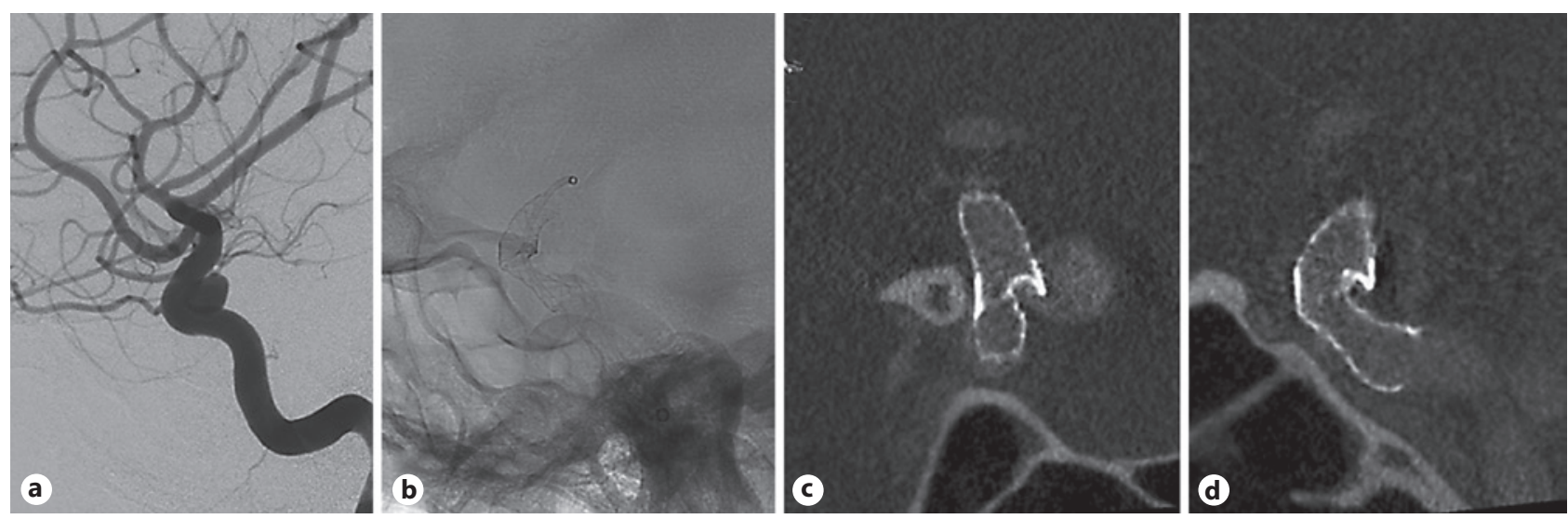

Fig. 2. a Case of a superior hypophyseal aneurysm treated with Pipeline (Medtronic). During deployment of the device, changes in the angulation of the vessel (b) caused the device to invaginate at the level of the neck of the aneurysm (c, d: oblique cross-sectional reconstruction of IACBCT).

CBCT with intra-arterial (IACBCT) injection has gained traction for intravascular image augmentation, allowing for optimized evaluation of these devices [3, 8]. IACBCT imaging modality provides details not only about the implanted device but also about the microvascular anatomy contributing to the decision making during these procedures $[3,7,8]$. Despite the multiple radiological advantages of the IACBCT, motion artifact even from subtle movements can significantly degrade the processed images; however, this was not a problem in our series since the images were acquired immediately after deployment, and therefore all patients were already under general procedure [3]. Additionally, this technique limits the evaluation of acute cerebral ischemia due to low sensitivity evaluating hypoattenuating lesions; however, we found that this was not a significant issue since hypoattenuation from ischemic changes would not be expected immediately after deployment of the devices [3]. Moreover, the IACBCT provides optimal visualization of luminal contrast, and one should be able to identify filling defects within the device as well as arterial occlusions [3, 5]. In our experience, IACBCT resulted in excellent qualitative visualization of the landing zones, 

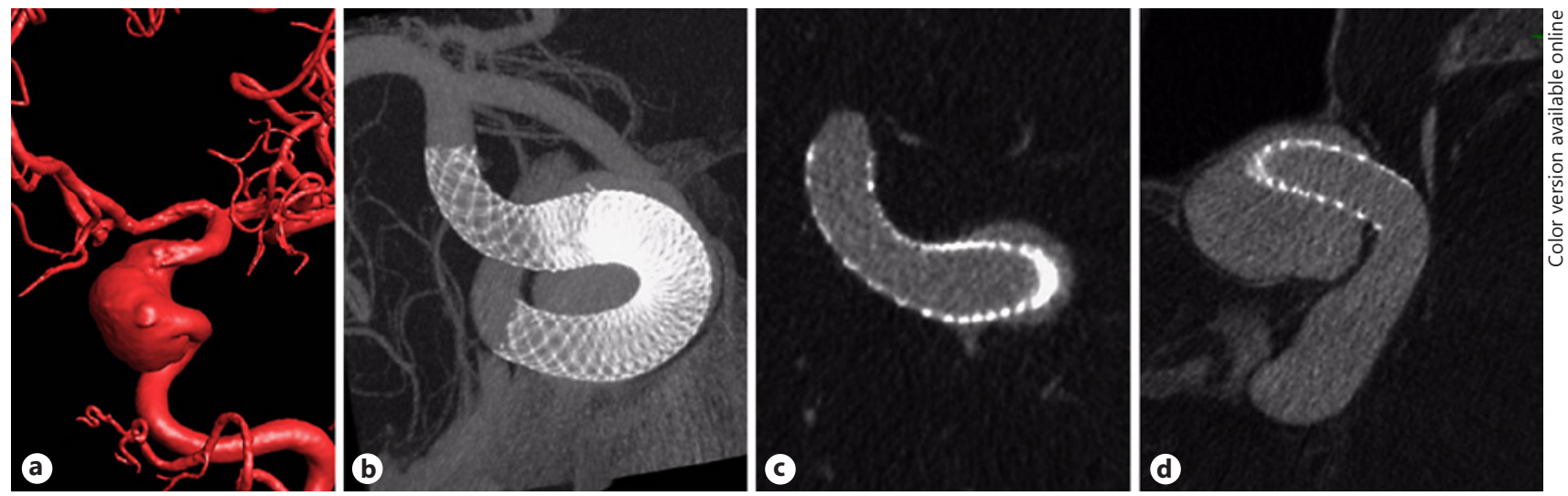

Fig. 3. a Case of a giant paraclinoid transitional aneurysm treated with a 2 Pipelines (Medtronic) construct. Reconstruction of IACBCT (b-d, cross-sectional) demonstrates perfect apposition of the distal end of the construct (c); however, the proximal end of the construct covers the proximal aspect of the neck by $1.5 \mathrm{~mm}$ (b and $\mathbf{d}$ ). Due to the risk of intra-aneurysmal sent migration, a third Pipeline was placed successfully. As predicted, during the navigation of the microcatheter for deployment of the third Pipeline, the proximal end of the initial stent construct migrated into the aneurysm.
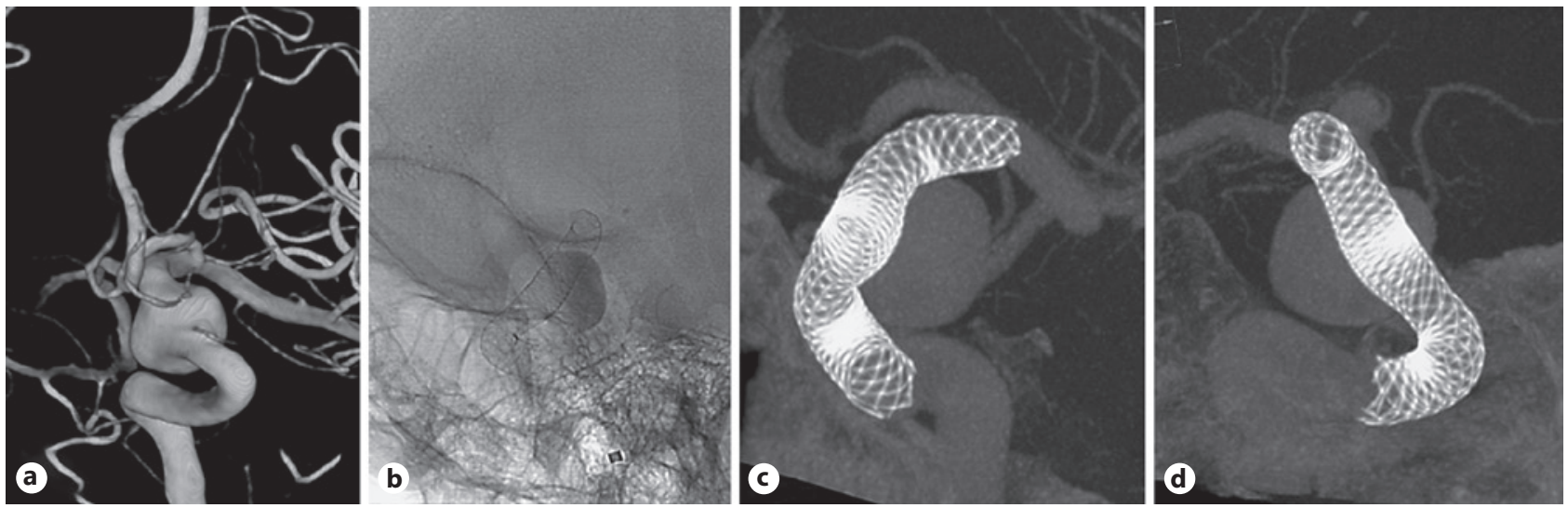

Fig. 4. Case of a large fusiform supraclinoid aneurysm (a) treated with Pipeline (Medtronic) (b-d). c, d Reconstruction of IACBCT demonstrates the intentional compaction of the cells/struts of the flow diverter at the midportion of the fusiform aneurysm.

conformability to the arterial geometry, presence of deformities, and wall apposition for both flow diverters.

Another advantage of this technique is the lower contrast agent dose [3]. IACBCT imaging modality is frequently obtained with a $10-20 \%$ diluted contrast [ 5,7$]$. A $14 \%$ dilution formula has been proposed for evaluation of stent-assisted coil embolization with CBCT [11]. One report recommends the use of $0.2 \mathrm{~cm}^{3} / \mathrm{s}$ contrast infusion rate and saline flush with a 300 $\mathrm{mm} \mathrm{Hg}$ pressure bag rather than a dilution formula within the power injector [7]. In this study, the CBCT contrast protocol included a $10-20 \%$ dilution resulting in good imaging quality of the parameters evaluated.

One significant advantage of IACBCT is that it can be performed intraoperatively, allowing for real-time intervention adjustment in accordance to image data, unlike multidetector CT and MRA [5, 8]. This is demonstrated in our case examples (Fig. 1-4). Early intraoperative 
recognition of potential problems related to the relationship between the flow diverter, the aneurysm neck, and the parent vessel can impact the clinical outcome by not only decreasing procedural and postprocedural risks but also increasing the effectiveness of the treatment [3, 8]. Incomplete stent expansion is one of the most important mechanisms related to thromboembolic events and thrombosis [4,5]. Moreover, malapposed stents could potentially result in an impaired reentry to previously stented vessels if subsequent vessel retreatment is required.

Visualization of a neurovascular stent with CT angiogram (CTA) and head MRA is limited by artifacts [3, 4, 8]. MRA is frequently degraded by metallic susceptibility artifacts [3]. Moreover, the neurovascular stents are not sufficiently radiopaque to be appropriately evaluated with conventional angiography $[3,5]$. Therefore, using the current imaging standards to determine the exact location, geometry, and wall apposition of these stents in relation to the host vasculature is challenging [8].

Patel et al. [5] utilized a standardized questionnaire, scoring cases for the quality of visualization of a stent and the parent vessels addressing not only wall apposition of the stent but also the presence of calcification or filling defect of the stented segment, and this was feasible in $73 \%$ of their cohort, although they had a low K statistic for agreement. The IACBCT imaging technique with our institutional protocol was demonstrated to be useful for the visualization of certain critical technical scenarios such as identifying an incomplete coverage of the aneurysm neck, a stent kinking, or the presence of an incomplete expansion and poor wall apposition. The above allowed further intervention and medical decision-making.

There are limitations to this study. First, this is a retrospective analysis and therefore, selection bias may be present; second, the data presented in this study were not independently adjudicated.

The intention of this study is to share our radiological experience in using this imaging modality during flow diverter device evaluations. We found this imaging protocol convenient as it provides valuable radiological information to the operator in the intraoperative setting of flow diverter device placement. This methodology could be applicable in other institutions as a useful tool during the periprocedural evaluation of flow diverter device placement.

\section{Disclosure Statement}

Dennys Reyes, MD, has no disclosures or conflict of interest. Victor Becerra has no disclosures or conflict of interest. Indiana Alcala, ARNP, has no disclosures or conflict of interest. Italo Linfante, MD, is a consultant, proctor, and speaker for Medtronic; consultant for Stryker; shareholder for Surpass and Three Rivers. Guilherme Dabus, MD, is a consultant, proctor, and speaker for Medtronic; shareholder for Surpass.

\section{References}

1 O'Kelly CJ, Krings T, Fiorella D, Marotta TR: A novel grading scale for the angiographic assessment of intracranial aneurysms treated using flow diverting stents. Interv Neuroradiol 2010;16:133-137.

2 Kamran M, Yarnold J, Grunwald IQ, Byrne JV: Assessment of angiographic outcomes after flow diversion treatment of intracranial aneurysms: a new grading schema. Neuroradiology 2011;53:501-508.

3 Ansari SA, Aoun SG, Bendok BR: Cone beam computed tomography in the neurointerventional room: beyond vessels. World Neurosurg 2012;77:659-661.

4 Tsuruta W, Matsumaru Y, Hamada Y, Hayakawa M, Kamiya Y: Analysis of closed-cell intracranial stent characteristics using cone-beam computed tomography with contrast material. Neurol Med Chir 2013;53:403408.

5 Patel NV, Gounis MJ, Wakhloo AK, Noordhoek N, Blijd J, Babic D, Takhtani D, Lee SK, Norbash A: Contrastenhanced angiographic cone-beam CT of cerebrovascular stents: experimental optimization and clinical application. AJNR Am J Neuroradiol 2011;32:137-144. 
6 Snoeren RM, Soderman M, Kroon JN, Roijers RB, de With PH, Babic D: High-resolution 3D X-ray imaging of intracranial nitinol stents. Neuroradiology 2012;54:155-162.

7 Jo KI, Kim SR, Choi JH, Kim KH, Jeon P: Contrast-enhanced angiographic cone-beam computed tomography without pre-diluted contrast medium. Neuroradiology 2015;57:1121-1126.

8 Kizilkilic O, Kocer N, Metaxas GE, Babic D, Homan R, Islak C: Utility of VasoCT in the treatment of intracranial aneurysm with flow-diverter stents. J Neurosurg 2012;117:45-49.

9 Becske T, Kallmes DF, Saatci I, McDougall CG, Szikora I, Lanzino G, Moran CJ, Woo HH, Lopes DK, Berez AL, Cher DJ, Siddiqui AH, Levy EI, Alburquerque FC, Fiorella DJ, Berentei Z, Marosfoi M, Cekirge SH, Nelson PK: Pipeline for uncoilable or failed aneurysms: results from a multicenter clinical trial. Radiology 2013;267:858-868.

10 Colby GP, Lin LM, Caplan JM, Jiang B, Michniewicz B, Huang J, Tamargo RJ, Coon AL: Flow diversion of large internal carotid artery aneurysms with the surpass device: impressions and technical nuance from the initial North American experience. J Neurointerv Surg 2016;8:279-286.

11 Kuriyama T, Sakai N, Niida N, Sueoka M, Beppu M, Dahmani C, Kojima I, Sakai C, Imamura H, Masago K, Katakami N: Dose reduction in cone-beam CT scanning for intracranial stent deployment before coil embolization of intracranial wide-neck aneurysms. Interv Neuroradiol 2016;22:420-425. 